\title{
Role of serum organic solute transporter alpha/beta and sodium taurocholate cotransporting polypeptide in intrahepatic cholestasis of pregnancy
}

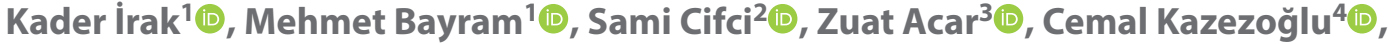 \\ Elif Yorulmaz ${ }^{5}$, Suleyman Yildirim² ${ }^{2}$ \\ ${ }^{1}$ Department of Gastroenterology, Kanuni Sultan Suleyman Training and Research Hospital, Istanbul, Turkey \\ ${ }^{2}$ Department of Gastroenterology with Başakşehir Cam and Sakura City Hospital, Istanbul, Turkey \\ ${ }^{3}$ Department of Gynecology and Obstetrics, Kanuni Sultan Suleyman Training and Research Hospital, Istanbul, Turkey \\ ${ }^{4}$ Department of Biochemistry, Kanuni Sultan Suleyman Training and Research Hospital, Istanbul, Turkey \\ ${ }^{5}$ Department of Gastroenterology, Bagcilar Training and Research Hospital, Istanbul, Turkey
}

\begin{abstract}
Objectives: This study aimed to assess the role of OST- $a$, OST- $\beta$ and NTCP in patients with ICP, with a view to determine patients with severe prognosis and to minimize adverse fetal outcomes.

Material and methods: Sixty-nine pregnant women diagnosed with ICP and 50 healthy women were included the study. Serum OST- $\alpha$, OST- $\beta$ and NTCP were measured using ELISA kits.

Results: The median OST-a levels were $176.3 \mathrm{pg} / \mathrm{mL}$ in women with ICP and $201 \mathrm{pg} / \mathrm{mL}$ in healthy subjects $(p=0.205)$. The median OST- $\beta$ levels were found to be $51.17 \mathrm{pg} / \mathrm{mL}$ in patients with ICP and $40.9 \mathrm{pg} / \mathrm{mL}$ in controls $(p=0.033)$. Median NTCP levels were $519.7 \mathrm{ng} / \mathrm{mL}$ in the ICP group and $483.3 \mathrm{ng} / \mathrm{mL}$ in healthy women $(p=0.051)$.

Conclusions: This is the first study to evaluate serum levels of OST- $a$, OST- $\beta$ and NTCP in patients with ICP. It is likely that OST- $\alpha$, OST- $\beta$ and NTCP contribute to the etiopathogenesis of ICP. Serum OST- $\alpha$ and OST- $\beta$ levels can be used as diagnostic and monitoring markers of ICP, and the inhibition of these molecules could provide therapeutic benefit in ICP by reducing the circulation of enterohepatic bile acids.

Key words: bile acids; intrahepatic cholestasis of pregnancy; organic solute transporter alpha/beta; sodium taurocholate cotransporting polypeptide
\end{abstract}

Ginekologia Polska 2021; 92, 11: 767-773

\section{INTRODUCTION}

Intrahepatic cholestasis of pregnancy (ICP) is the most common pregnancy-specific liver disease characterized by elevated serum bile acids, maternal pruritus in the second half of pregnancy, and an increased rate of adverse fetal outcomes, including intrapartum fetal distress, spontaneous preterm delivery, meconium staining of the amniotic fluid, and even stillbirths or intrauterine fetal death [1]. Although maternal prognosis is excellent, the risk of complications to the fetus and its development increases in parallel with maternal blood levels of total bile acids (TBA) [2]. After delivery, the symptoms and biochemical abnormalities usually resolve rapidly - almost spontaneously. The incidence of
ICP is estimated to range between $1 \%$ and $4 \%$, but varies greatly depending on environmental factors, ethnic origin and genetic predisposition [3]. The etiopathogenesis of the disease is not fully understood, but is probably multifactorial with genetic, environmental, dietary and hormonal backgrounds that also effect its severity [4]. The impairment of the transport, excretion and function of bile acids may be associated with the development of ICP.

Organic solute transporter alpha/beta (OST- $\alpha /$ OST- $\beta$ ) are solute carrier proteins that were identified in the hepatocyte and intestinal epithelium. These heteromeric transporters, which are located in basolateral membranes, are critical in the reabsorption and enterohepatic circulation of ster-

\section{Corresponding author:}

Kader Irak

Department of Gastroenterology, Kanuni Sultan Suleyman Training and Research Hospital, 34303 Istanbul, Turkey

e-mail: drkaderirak@hotmail.com 
oid-based molecules, particularly bile acids [5]. Furthermore, it was shown in a mice study that the functions of OST- $\alpha$ and OST- $\beta$ include the protection of the ileal epithelium against the accumulation of bile acids [6]. The expression of OST- $\alpha$ and OST $\beta$ proteins were found to be altered in conditions that cause elevation in bile acids, including extrahepatic cholestasis, primary biliary cholangitis and nonalcoholic steatohepatitis [5]. However, to our knowledge, there are no studies that have investigated serum levels of OST- $\alpha /$ OST- $\beta$ in patients with ICP.

Sodium taurocholate cotransporting polypeptide (NTCP) is another transport protein localized in the basolateral membrane of the hepatocyte. Its primary function is to collect conjugated bile salts from the circulation via cotransport of sodium [7]. NTCP has extremely high affinity and specificity for bile acids, and is reported to recover up to $90 \%$ from the circulation [3]. The gene encoding for NTCP (Solute Carrier Family 10 Member 1, SLC10A1) has been shown to have biallelic mutations that impair NTCP function; subsequently leading to increased serum levels of TBA [8]. In a recent case study including two women who had suffered from ICP, researchers found that homozygote mutations of SLC10A1 can lead to ICP development [7]. To date, the results of studies examining the role of NTCP in ICP patients remain rather limited. However, no studies have examined the serum levels of NTCP in ICP patients.

The aim of this study was to evaluate the serum levels of OST- $\alpha$ /OST- $\beta$ and NCPT in patients with ICP with a view to identify whether they can be associated with disease development and/or severity.

\section{MATERIAL AND METHODS}

This was a prospective single center study that was carried out between July 2020 and September 2020 at the Maternal-Fetal Medicine Unit of Health Sciences University Kanuni Sultan Suleyman Research and Training Hospital, Istanbul, Turkey. A total of 69 pregnant women diagnosed with ICP and 50 healthy women with completely normal pregnancies (as the control group) in the third trimester were included in the study. The diagnosis of ICP was based on the following criteria: (i) presence of pruritus that cannot be explained with any other condition, (ii) elevated levels of serum aminotransferase ( $>40 \mathrm{IU} / \mathrm{L}$ ) and (iii) elevated levels of serum fasting TBA ( $>10 \mu \mathrm{mol} / \mathrm{L}$ ). ICP severity was defined according to TBA levels as: mild (10-40 $\mu \mathrm{mol} / \mathrm{L})$ and severe ( $>40 \mu \mathrm{mol} / \mathrm{L}$ ). Participants with a history of conditions that cause abnormalities in liver function tests, including hepatitis, gallstones or gallbladder diseases, acute fatty liver of pregnancy, hepatotoxic drug consumption, primary biliary cholangitis, primary sclerosing cholangitis, active infection and chronic inflammatory conditions, were excluded from the study. Additionally, patients with gestational diabetes, preeclampsia, gestational thyroid disorders, malignancy, thrombophilia, those who had pruritus with normal bile acids, and those with skin lesions were excluded.

Gestational age was calculated according to last menstruation date and first trimester obstetric radiologic examination. Each ICP patient completed a questionnaire rating the severity of pruritus on a numerical scale from 1 to 10. Scores ranging from 1 to 4 were defined as 'mild', 4 to 7 as 'moderate', and a score of 8 or higher was defined as 'severe' pruritus. Patient characteristics, including maternal age, gravidity, parity, gestational age at the time of diagnosis, type of delivery, birth weight of neonates, and length of neonatal intensive care unit stay were recorded from patient files. The study protocol was approved by the Research Ethics Committee of Kanuni Sultan Suleyman Training and Research Hospital. All research procedures were conducted based on the ethical standards of the Declaration of Helsinki. Written and verbal informed consent was obtained from participating women prior to participation and sampling.

Blood samples were collected from all participants during the third trimester (at the time of initial admission) before the administration of medications or interventions. Hemogram (complete blood count) and routine blood biochemistry values were examined within one hour after sampling. For further analysis of OST- $\alpha$, OST- $\beta$ and NTCP, extracted serum samples (centrifuged at $4^{\circ} \mathrm{C}$ and 3000 RPM for $10 \mathrm{~min}$ utes) were frozen at $-80^{\circ} \mathrm{C}$ until measurements. Hemogram parameters, including white blood cell count (WBC), neutrophil (NEU) and lymphocyte (LYM) counts, hemoglobin value $(\mathrm{Hb})$, platelet count $(\mathrm{PLT})$, mean platelet volume (MPV) were measured with a CELL-DYN 3700 System (Abbott Laboratories, IL, USA). Neutrophil to lymphocyte ratio (NLR) and Platelet to lymphocyte ratio (PLR) were calculated. Blood biochemistry parameters, including alanine aminotransferase (ALT), aspartate aminotransferase (AST), total bilirubin, direct bilirubin and TBA levels were determined with spectrophotometric methods using an Abbott Architect c8000 autoanalyzer (Abbott Laboratories, IL, USA). The serum OST- $\alpha$ and OST- $\beta$ levels were measured using enzyme-linked immunosorbent assay (ELISA) kits (Elabscience Biotechnology, USA) as per the manufacturer's instructions. The serum NTCP concentrations were determined with a commercially available Human Sodium Bile Acid Cotransporter/NTCP ELISA Kit (Abbexa Ltd, Cambridge, UK). The measurement range was $0.78-50 \mathrm{ng} / \mathrm{mL}$ and the assay sensitivity was $<0.29 \mathrm{ng} / \mathrm{mL}$. The intra-assay and inter-assay variations were $<10 \%$ and $<12 \%$, respectively.

\section{Statistical analysis}

All analyses were performed on SPSS v21 (SPSS Inc., Chicago, IL, USA). For the normality check, the Kolmogorov-Smirnov test with Lilliefors correction was used. Data are 
given as mean \pm standard deviation or median ( $1{ }^{\text {st }}$ quartile$-3^{\text {rd }}$ quartile) for continuous variables according to normality of distribution, and as frequency (percentage) for categorical variables. Normally distributed variables were analyzed with the independent samples t test. Non-normally distributed variables were analyzed with the Mann Whitney $U$ test. Categorical variable distributions were analyzed with Chi-square tests. Repeated (paired) measurements were analyzed with the Wilcoxon signed ranks test. Spearman correlation coefficients were calculated to evaluate relationships between continuous variables. Diagnostic performance of variables were evaluated by Receiver Operating Characteristic (ROC) curve analysis. $p<0.05$ values were defined as statistically significant.

\section{RESULTS}

Sixty-nine patients with ICP and 50 healthy women were included in the study. The mean age was $29.8 \pm 5.8$ years in the study group and $29 \pm 5.7$ years in the control group $(p=0.468)$. The serum levels of ALT, AST, total and direct bilirubin were higher in the patient group (all, $p<0.05$ ). Elevated serum levels of hemoglobin, platelet count and PLR were found in patients with ICP compared to control subjects (all, $p<0.05$ ). No differences were found between the two groups with regard to WBC, MPV, RDW, NLR, neutrophil and lymphocyte counts (all, $p>0.05$ ). Demographic characteristics and laboratory values of the patient and the control groups are shown in Table 1.

The median OST-a levels were $176.32 \mathrm{pg} / \mathrm{mL}$ in women with ICP and $201.03 \mathrm{pg} / \mathrm{mL}$ in healthy subjects $(p=0.205)$. Median OST- $\beta$ levels were found to be significantly higher $(51.17 \mathrm{pg} / \mathrm{mL})$ in patients with ICP compared to controls (40.90 pg/mL) ( $p=0.033)$. The median NTCP values were $519.71 \mathrm{ng} / \mathrm{mL}$ in the ICP and $483.34(\mathrm{ng} / \mathrm{mL})$ in the healthy control group ( $p=0.051$ ) (Tab. 1). ROC analysis demonstrated that OST- $\beta$ levels had $53.62 \%$ sensitivity, $73.47 \%$ specific-

\begin{tabular}{|c|c|c|c|}
\hline & Controls $(n=50)$ & Patients $(n=69)$ & $p$ value \\
\hline Age [years] & $29.82 \pm 5.81$ & $29.04 \pm 5.70$ & 0.468 \\
\hline Gestational week & $28(24-35)$ & $33(31-35)$ & 0.013 \\
\hline \multicolumn{4}{|l|}{ Numbers of pregnancy } \\
\hline 1 & $10(20.00 \%)$ & $21(30.43 \%)$ & \multirow{4}{*}{0.372} \\
\hline 2 & $13(26.00 \%)$ & $21(30.43 \%)$ & \\
\hline 3 & $14(28.00 \%)$ & $12(17.39 \%)$ & \\
\hline$\geq 4$ & $13(26.00 \%)$ & $15(21.74 \%)$ & \\
\hline ALT [IU/mL] & $9(8-13)$ & $72(48-152.5)$ & $<0.001$ \\
\hline AST [IU/mL] & $14.5(13-17)$ & $55(35-91)$ & $<0.001$ \\
\hline Total bilirubin [mg/dL] & $0.22(0.16-0.30)$ & $0.49(0.32-0.73)$ & $<0.001$ \\
\hline Direct bilirubin [mg/dL] & $0.11(0.10-0.12)$ & $0.27(0.18-0.50)$ & $<0.001$ \\
\hline $\mathrm{WBC}\left[\times 10^{3} / \mathrm{mm}^{3}\right]$ & $9.09 \pm 2.57$ & $9.49 \pm 1.89$ & 0.327 \\
\hline Hemoglobin [g/dL] & $11.15 \pm 1.13$ & $11.66 \pm 1.31$ & 0.030 \\
\hline $\mathrm{MPV}\left[\mu \mathrm{m}^{3}\right]$ & $10.85(10.1-11.9)$ & $11.15(10.2-11.7)$ & 0.618 \\
\hline RDW [\%] & $13.25(13-13.7)$ & $13.1(12.6-13.8)$ & 0.494 \\
\hline Neutrophil $\left[\times 10^{3} / \mathrm{mm}^{3}\right]$ & $7.79(6.39-8.4)$ & $7(5.3-7.8)$ & 0.054 \\
\hline Lymphocyte $\left[\times 10^{3} / \mathrm{mm}^{3}\right]$ & $1.9(1.7-2.3)$ & $1.8(1.6-2)$ & 0.051 \\
\hline Platelet $\left[\times 10^{3} / \mathrm{mm}^{3}\right]$ & $214.04 \pm 47.58$ & $245.87 \pm 74.17$ & 0.005 \\
\hline NLR & $3.85(3.18-4.51)$ & $3.91(2.83-4.68)$ & 0.907 \\
\hline PLR & $114.45(91.58-131.30)$ & $124.41(108.23-176.03)$ & 0.006 \\
\hline OST-alpha [pg/mL] & $201.03(167.01-238.36)$ & $176.32(145.76-212.27)$ & 0.205 \\
\hline OST-beta [pg/mL] & $40.90(30.62-51.16)$ & $51.17(36.44-62.42)$ & 0.033 \\
\hline NTCP $[n g / m L]$ & $483.34(98.23-601.82)$ & $519.71(416.58-677.62)$ & 0.051 \\
\hline
\end{tabular}

ALT — Alanine aminotransferase; AST — Aspartate aminotransferase; WBC — White blood cell counts; MPV — Mean platelet volume; NLR — Neutrophil to lymphocyte ratio; PLR — Platelet to lymphocyte ratio; OST-alpha — Organic solute transporter alpha; OST-beta — Organic solute transporter beta; NTCP — Sodium taurocholate cotransporting polypeptide; Data are given as mean \pm standard deviation or median ( $1^{\text {st }}$ quartile- $3^{\text {rd }}$ quartile) for continuous variables according to normality of distribution and as frequency (percentage) for categorical variables 
ity and $61.86 \%$ accuracy to determine presence of ICP with a cut-off point of 50.5 (AUC $=0.616,95 \%$ Cl: $0.513-0.718$ ) (data not shown).

The median TBA levels were $26(16-48) \mu \mathrm{mol} / \mathrm{L}$ in the patient group. According to TBA levels, 45 patients had mild ICP and 24 patients had severe ICP. Forty patients received 4 doses of ursodeoxycholic acid (UDCA) treatment, while 26 patients were treated with 3 doses. Following the administration of UDCA, 45 patients had reduction in pruritus complaints and 34 patients showed decreased ALT and AST levels. After deliveries, 17 of the neonates were admitted to the neonatal ICU (Tab. 2).

Correlation analyses between patient characteristics and laboratory results are shown in Table 3. In correlation analyses, we found negative correlations between maternal age and the levels of OST- $a$ and NTCP. A positive correlation was shown between NTCP and total bilirubin (Tab. 3).

\section{Table 2. Patients' characteristics and laboratory results}

\begin{tabular}{|c|c|}
\hline Total Bile acid [ $\mu \mathrm{mol} / \mathrm{L}]$ & $26(16-48)$ \\
\hline Patients with mild ICP $[<40]$ & $45(65.22 \%)$ \\
\hline Patients with severe ICP $[\geq 40]$ & $24(34.78 \%)$ \\
\hline UDCA & $4(3-4)$ \\
\hline 0 & $1(1.45 \%)$ \\
\hline 2 & $1(1.45 \%)$ \\
\hline 3 & $26(37.68 \%)$ \\
\hline 4 & $40(57.97 \%)$ \\
\hline 6 & $1(1.45 \%)$ \\
\hline \multicolumn{2}{|l|}{ Pruritus } \\
\hline Mild & $25(37.68 \%)$ \\
\hline Moderate & 19 (27.54\%) \\
\hline Severe & $24(34.78 \%)$ \\
\hline Decrease in pruritus after UDCA & $45(77.59 \%)$ \\
\hline Decrease in ALT after UDCA & 34 (73.91\%) \\
\hline Decrease in AST after UDCA & $34(72.34 \%)$ \\
\hline Alkaline phosphotase [IU/L] & $199.27 \pm 71.43$ \\
\hline Gamma glutamyl transferase [IU/L] & $17(11-29)$ \\
\hline Lactate dehydrogenase [IU/L] & $227.22 \pm 49.25$ \\
\hline Gestational week at birth & $37(36-38)$ \\
\hline \multicolumn{2}{|l|}{ Birth type } \\
\hline Vaginal & $22(37.93 \%)$ \\
\hline Cesarean & $36(62.07 \%)$ \\
\hline Weight at birth [grams] & $2793.17 \pm 581.35$ \\
\hline $\begin{array}{l}\text { The number of patients requiring ICU } \\
\text { admission }\end{array}$ & $17(29.31 \%)$ \\
\hline
\end{tabular}

ICP — INntrahepatic cholestasis of pregnancy; UDCA — Ursodeoxycholic acid; ICU - Intensive care unit; Data are given as mean \pm standard deviation or median ( $1^{\text {st }}$ quartile- $3^{\text {rd }}$ quartile) for continuous variables according to normality of distribution and as frequency (percentage) for categorical variables

\section{DISCUSSION}

This study was aimed at determining circulating levels of OST- $\alpha$, OST- $\beta$ and NTCP in patients with ICP order to identify whether these measures could be utilized to diagnose ICP, determine prognosis, or assess fetal outcomes. This was the first study to determine the serum levels of OST- $\alpha$, OST- $\beta$ and NTCP in patient with ICP. We demonstrated that serum OST- $\beta$ levels were significantly higher in patients with ICP compared to healthy individuals. We also observed higher NTCP levels in patients, but statistical significance was not present with a $p$ value of 0.051 .

Intrahepatic cholestasis of pregnancy has a complex and multifaceted etiology, involving complex interactions between genetic features, endocrine and metabolic disturbances, and environmental factors [4]. Altered secretion and transport of bile acids and other biliary lipids play an important role on the etiology of ICP [9]. Bile salts are the most common organic solutes in the bile [1]. Bile acids (cholic acid and chenodeoxycholic acid) are synthesized from cholesterol in the liver, conjugated with either taurine or glycine to form bile salts, and are exported into the biliary canaliculi via specific transport proteins [10]. When the bile acids reach the gastrointestinal tract, they maintain their emulsifying functions as physiological detergents to break down and encapsulate lipids and fat-soluble vitamins for absorption and transport. Bile acids are also metabolic signaling molecules with systemic endocrine effects [11]. The specific nuclear receptors of bile acids include, farnesoid $X$ receptor $(F X R)$, vitamin $D$ receptor, and the cell surface G protein-coupled bile acid receptor (GPBAR, TGR-5) [12]. After contributing to intestinal digestion and absorption, bile acids are actively transported into intestinal cells in the terminal ileum. The transporters involved in this function include OST- $\alpha$ and OST- $\beta$ [12] which transport the bile acids across the basolateral membrane of the enterocytes, followed by secretion into the portal circulation. They are actively transported back to hepatocytes via NTCP and organic anion transporting polypeptides [10]. The mutations and variations in genes encoding hepatobiliary transport proteins have been shown to cause abnormal bile secretion and increased circulatory bile acids in patient with ICP [13].

The OST- $\alpha$ and OST- $\beta$ heteromeric transport proteins are present in ileal enterocytes, cholangiocytes and the renal proximal tubule [14]. FXR mechanisms have been associated with the key regulatory roles of OST- $\alpha$ and OST- $\beta$ in maintaining homeostasis of bile acids [15]. As such, intestinal FXR induces the expression of OST- $\alpha$ and OST- $\beta$ as an adaptive response to cholestasis [12]. Boyer et al. demonstrated that hepatic OST- $\alpha$ and OST- $\beta$ mRNA levels were elevated by three-fold and 32-fold, respectively, in patients with primary biliary cirrhosis (PBC). They also demonstrated that OST-a and OST- $\beta$ expression increased in the livers of rats and mice 


\begin{tabular}{|c|c|c|c|c|}
\hline & & OST-alpha & OST-beta & NTCP \\
\hline \multirow{2}{*}{ Age } & $r$ & $-0.278^{*}$ & -0.169 & $-0.277^{*}$ \\
\hline & $p$ & 0.002 & 0.067 & 0.002 \\
\hline \multirow{2}{*}{ Gestational week } & $r$ & 0.056 & -0.105 & 0.065 \\
\hline & $\mathrm{p}$ & 0.548 & 0.259 & 0.483 \\
\hline \multirow{2}{*}{ Total Bile acid } & $r$ & -0.177 & -0.099 & -0.107 \\
\hline & $p$ & 0.145 & 0.417 & 0.381 \\
\hline \multirow{2}{*}{ ALT } & $r$ & -0.150 & 0.144 & 0.133 \\
\hline & $\mathrm{p}$ & 0.106 & 0.121 & 0.152 \\
\hline \multirow{2}{*}{ AST } & $r$ & -0.117 & 0.145 & 0.115 \\
\hline & $\mathrm{p}$ & 0.213 & 0.120 & 0.218 \\
\hline \multirow{2}{*}{ Total bilirubin } & $r$ & -0.058 & 0.068 & $0.236^{*}$ \\
\hline & $p$ & 0.540 & 0.472 & 0.012 \\
\hline \multirow{2}{*}{ Direct bilirubin } & $r$ & -0.045 & 0.182 & 0.156 \\
\hline & $p$ & 0.632 & 0.052 & 0.098 \\
\hline \multirow{2}{*}{$\begin{array}{l}\text { Alkaline } \\
\text { phosphatase }\end{array}$} & $r$ & 0.168 & -0.056 & 0.028 \\
\hline & $p$ & 0.203 & 0.673 & 0.831 \\
\hline \multirow{2}{*}{$\begin{array}{l}\text { Gamma-glutamyl } \\
\text { transferase }\end{array}$} & $r$ & 0.024 & -0.020 & -0.006 \\
\hline & $\mathrm{p}$ & 0.858 & 0.878 & 0.961 \\
\hline \multirow{2}{*}{$\begin{array}{l}\text { Lactate } \\
\text { Dehydrogenase }\end{array}$} & $r$ & 0.238 & -0.100 & 0.053 \\
\hline & $p$ & 0.083 & 0.471 & 0.703 \\
\hline \multirow{2}{*}{ WBC } & $r$ & 0.102 & 0.128 & 0.063 \\
\hline & $p$ & 0.274 & 0.169 & 0.503 \\
\hline \multirow{2}{*}{$\begin{array}{l}\text { Hemoglobin } \\
\text { value }\end{array}$} & $r$ & -0.033 & 0.130 & 0.150 \\
\hline & $p$ & 0.724 & 0.163 & 0.107 \\
\hline \multirow{2}{*}{ MPV } & $r$ & -0.107 & -0.033 & 0.090 \\
\hline & $p$ & 0.250 & 0.728 & 0.332 \\
\hline \multirow{2}{*}{ RDW } & $r$ & 0.010 & -0.077 & -0.011 \\
\hline & $p$ & 0.911 & 0.408 & 0.909 \\
\hline \multirow{2}{*}{$\begin{array}{l}\text { Neutrophil } \\
\text { counts }\end{array}$} & $r$ & -0.005 & 0.076 & 0.105 \\
\hline & $p$ & 0.957 & 0.418 & 0.261 \\
\hline \multirow{2}{*}{$\begin{array}{l}\text { Lymphocyte } \\
\text { count }\end{array}$} & $r$ & 0.010 & 0.041 & 0.010 \\
\hline & $p$ & 0.916 & 0.659 & 0.918 \\
\hline \multirow{2}{*}{$\begin{array}{l}\text { Platelet } \\
\text { count }\end{array}$} & $r$ & -0.027 & 0.177 & -0.059 \\
\hline & $p$ & 0.774 & 0.057 & 0.527 \\
\hline \multirow{2}{*}{ NLR } & $r$ & -0.012 & 0.044 & 0.125 \\
\hline & $p$ & 0.899 & 0.641 & 0.181 \\
\hline \multirow{2}{*}{ PLR } & $r$ & -0.018 & 0.124 & -0.056 \\
\hline & $p$ & 0.845 & 0.181 & 0.547 \\
\hline
\end{tabular}

ALT — Alanine aminotransferase; AST — Aspartate aminotransferase; WBC — White blood cell counts; MPV — Mean platelet volume; NLR — Neutrophil to lymphocyte ratio; PLR — Platelet to lymphocyte ratio; OST-alpha — Organic solute transporter alpha; OST-beta — Organic solute transporter beta; NTCP — Sodium taurocholate cotransporting polypeptid. $r$ - Spearman correlation coefficient; ${ }^{*}$ Correlation is significant at the 0.05 level

following common bile duct ligation [16]. In another study, Schaap et al. [17], showed increased OST- $a$ and OST- $\beta$ mRNA levels in the livers of patients with extrahepatic cholestasis. Malinen and colleagues [18] identified significantly in- creased expression and protein levels of OST- $\beta$ in livers from patients with nonalcoholic steatohepatitis (NASH) and PBC. They also observed unchanged OST-a mRNA expression and higher OST-a protein levels in patients with NASH and 
PBC when compared to healthy subjects (but statistical significance was not present). Chai et al. [19], revealed in 22 liver samples from cholestatic patients with biliary obstruction (due to gallstones) that mRNA and protein levels of OST- $\beta$ protein levels was significantly increased ( $10.4 \mathrm{fold}$ ), while OST-a protein levels were decreased while transcription was unchanged compared to control livers. Soroka and colleagues [20] demonstrated in mice with genetically deficient OST-a that loss of OST-a prevents liver damage in obstructive cholestasis through adaptive responses in both the kidney and liver. In agreement with these studies, we found significantly elevated serum OST- $\beta$ levels and somewhat decreased OST-a level (no statistical significance) in patients with ICP. Our results may suggest that OST- $a$ and OST- $\beta$ are induced in ICP, possibly due their association with the re-uptake of bile acids. The increase in OST- $\beta$ levels may be related to the elevation of intrahepatic bile acid and TBA concentrations during cholestasis. Further studies are necessary to determine whether serum levels of OST- $\beta$ can be used as a marker for the diagnosis and prognostic assessment of ICP.We demonstrated a cut-off point of $>50.5$ for the identification of ICP with OST- $\beta$ levels. Our results suggest that OST- $\alpha$ and OST- $\beta$ could be contributing factors in the pathogenesis of ICP. However, there is still uncertainty about the contribution of OST- $\alpha$ and OST- $\beta$ to bile acid efflux and attenuation of cholestasis in literature [21].

NTCP is a sodium-dependent primary transporter that transports conjugated bile salts from the plasma to the hepatocyte. NTCP is highly expressed in the basolateral membrane of hepatocytes and plays an important role in the maintenance of enterohepatic circulation of bile salts [7]. Vaz et al. [22], demonstrated mild hypotonia, growth retardation, and elevated total bile acid levels (> $1500 \mu \mathrm{M})$ without signs of cholestatic jaundice, pruritus, or liver dysfunction in a patient with NTCP deficiency caused by a point mutation in the SLC10A1 gene. Kojima et al. [23], showed no change in NTCP expression in patients with chronic hepatitis $C$ or primary biliary cirrhosis stage I and II, while they identified downregulation in stage III. A case reported by Van Herpe et al. [24], described a woman with ICP who had lost her fetus at 38 weeks of pregnancy due to spontaneous rupture of membranes with meconium-stained fluids. They revealed persistently elevated TBA levels and NTCP deficiency in the post-partum period. In another clinical study, Chen et al. [7], demonstrated elevated TBA levels in five patients with NTCP deficiency diagnosed with SLC10A1 gene analysis. They also showed that two of these individuals had suffered from ICP without intense pruritus in the third trimester of pregnancy - leading to caesarean section delivery in one. In our study, we found higher NTCP levels in the ICP group compared to controls, a result which was only marginally insignificant $(p=0.051)$. This indicates that elevated NTCP levels may be associated with increased bile acid uptake into the liver in ICP disease. Furthermore, NTCP was correlated with age and total bilirubin levels. Consistent with our findings, Sargiacomo et al. [25], found that NTCP protein levels were modulated in an age-dependent manner in the hepatocyte membrane. In addition, Shneider et al. [26], demonstrated that NTCP mRNA levels correlated inversely with serum bilirubin in patients with pre- and postportoenterostomy biliary atresia. A mild increase in bilirubin levels in ICP patients can lead to increased levels of transport protein for hepatic uptake, and thus the excretion of bile salts and bilirubin.

Several limitations of the current work should be mentioned. First, the study had a relatively small sample size which may have reduced statistical strength; thus future studies will benefit from utilizing the results of this study in their power analyses. Secondly, the study was limited to Turkish ICP patients; considering the known genetic and environmental variations in the characteristics of patients with ICP, our results may not be generalized for women in other geographic regions. Thirdly, we were not able to evaluate fetal cord levels or placental levels of these biomarkers. Lastly, the lack of mRNA expression measurements may also be considered as a limitation.

\section{CONSLUSIONS}

In conclusion, this is the first study to evaluate serum levels of OST- $\alpha$, OST- $\beta$ and NTCP in patients with ICP. We demonstrated significantly elevated OST- $\beta$ levels in patients with ICP. We believe that OST- $\beta$ and NTCP could contribute to the etiopathogenesis of ICP. Early identification and management of ICP prevents adverse outcomes, especially for the fetus. It is crucial to determine whether serum levels of OST- $\alpha$, OST- $\beta$ or NTCP can be used as diagnostic or monitoring markers. Furthermore, considering their role in bile acid turnover, it may be interesting to evaluate the inhibition of these transporters as a possible therapeutic option in ICP.

\section{Source of Funding}

This research did not receive any specific grant from funding agencies in the public, commercial, or not-for-profit sectors

\section{Conflict of interest}

Conflicts of interest relevant to this article was not reported.

\section{REFERENCES}

1. Kumar S, Puri P, Gujral K. Intrahepatic cholestasis of pregnancy. Current Medicine Research and Practice. 2018; 8(6): 230-234, doi: 10.1016/j. cmrp.2018.11.006.

2. Celik S, Calışan CS, CelikH, et al. Predictors of adverse perinatal outcomes in intrahepatic cholestasis of pregnancy. Ginekol Pol. 2019; 90(4): 217-222, doi: 10.5603/GP.2019.0039, indexed in Pubmed: 31059115. 
3. Smith DD, Rood KM. Intrahepatic Cholestasis of Pregnancy. Clin Obstet Gynecol. 2020; 63(1): 134-151, doi: 10.1097/GRF.0000000000000495, indexed in Pubmed: 31764000.

4. Piechota J, Jelski W. Intrahepatic Cholestasis in Pregnancy: Review of the Literature. J Clin Med. 2020; 9(5), doi: 10.3390/jcm9051361, indexed in Pubmed: 32384779.

5. Beaudoin JJ, Brouwer KLR, Malinen MM. Novel insights into the organic solute transporter alpha/beta, OSTa/ $\beta$ : From the bench to the bedside. Pharmacol Ther. 2020; 211: 107542, doi: 10.1016/j. pharmthera.2020.107542, indexed in Pubmed: 32247663.

6. Ferrebee CB, Li J, Haywood J, et al. Organic Solute Transporter $\alpha-\beta$ Protects Ileal Enterocytes From Bile Acid-Induced Injury. Cell Mol Gastroenterol Hepatol. 2018; 5(4):499-522, doi: 10.1016/j.jcmgh.2018.01.006, indexed in Pubmed: 29930976.

7. Chen R, Deng M, Rauf YM, et al. Intrahepatic Cholestasis of Pregnancy as a Clinical Manifestation of Sodium-Taurocholate Cotransporting Polypeptide Deficiency. Tohoku J Exp Med. 2019; 248(1): 57-61, doi: 10.1620/tjem.248.57, indexed in Pubmed: 31142693.

8. Karpen SJ, Dawson PA. Not all (bile acids) who wander are lost: the first report of a patient with an isolated NTCP defect. Hepatology. 2015; 61(1): 24-27, doi: 10.1002/hep.27294, indexed in Pubmed: 24995605.

9. Tayyar AT, Tayyar A, Kozali S, et al. Evaluation of FGF-19 and $\beta$-klotho as biomarkers in patients with intrahepatic cholestasis of pregnancy. Arch Med Sci. 2019; 15(1): 113-119, doi: 10.5114/aoms.2017.72424, indexed in Pubmed: 30697260.

10. Di Ciaula A, Garruti G, Lunardi Baccetto R, et al. Bile Acid Physiology. Ann Hepatol. 2017; 16(Suppl. 1: s3-105.): s4-ss14, doi: 10.5604/01.3001.0010.5493, indexed in Pubmed: 29080336.

11. Molinaro A, Wahlström A, Marschall HU. Role of Bile Acids in Metabolic Control. Trends Endocrinol Metab. 2018; 29(1): 31-41, doi: 10.1016/j. tem.2017.11.002, indexed in Pubmed: 29195686.

12. Chiang JYL, Ferrell JM. Bile Acid Biology, Pathophysiology, and Therapeutics. Clin Liver Dis (Hoboken). 2020; 15(3): 91-94, doi: 10.1002/cld.861, indexed in Pubmed: 32257118.

13. Tayyar A, Temel Yuksel I, Koroglu N, et al. Maternal copeptin levels in intrahepatic cholestasis of pregnancy. J Matern Fetal Neonatal Med. 2018; 31(15): 2066-2070, doi: 10.1080/14767058.2017.1335708, indexed in Pubmed: 28552021.

14. Ballatori N, Christian WV, Lee JY, et al. OSTalpha-OSTbeta: a major basolateral bile acid and steroid transporter in human intestinal, renal, and biliary epithelia. Hepatology. 2005; 42(6): 1270-1279, doi: 10.1002/hep.20961, indexed in Pubmed: 16317684.

15. Boyer JL. OSTa-OST $\beta$ Guards the lleal Enterocyte From the Accumulation of Toxic Levels of Bile Acids. Cell Mol Gastroenterol Hepatol. 2018; 5(4): 649-650, doi: 10.1016/j.jcmgh.2018.01.015, indexed in Pubmed: 29713669.
16. Boyer JL, Trauner M, Mennone A, et al. Upregulation of a basolateral FXR-dependent bile acid efflux transporter OSTalpha-OSTbeta in cholestasis in humans and rodents. Am J Physiol Gastrointest Liver Physiol. 2006; 290(6): G1124-G1130, doi: 10.1152/ajpgi.00539.2005, indexed in Pubmed: 16423920.

17. Schaap FG, van der Gaag NA, Gouma DJ, et al. High expression of the bile salt-homeostatic hormone fibroblast growth factor 19 in the liver of patients with extrahepatic cholestasis. Hepatology. 2009; 49(4): 1228-1235, doi: 10.1002/hep.22771, indexed in Pubmed: 19185005.

18. Malinen MM, Ali I, Bezençon J, et al. Organic solute transporter OSTa/ $\beta$ is overexpressed in nonalcoholic steatohepatitis and modulated by drugs associated with liver injury. Am J Physiol Gastrointest Liver Physiol. 2018; 314(5): G597-G609, doi: 10.1152/ajpgi.00310.2017, indexed in Pubmed: 29420067.

19. Chai J, Feng $X$, Zhang $L$, et al. Hepatic expression of detoxification enzymes is decreased in human obstructive cholestasis due to gallstone biliary obstruction. PLoS One. 2015; 10(3): e0120055, doi: 10.1371/journal.pone.0120055, indexed in Pubmed: 25798860.

20. Soroka CJ, Mennone A, Hagey LR, et al. Mouse organic solute transporter alpha deficiency enhances renal excretion of bile acids and attenuates cholestasis. Hepatology. 2010; 51(1): 181-190, doi: 10.1002/hep.23265, indexed in Pubmed: 19902485.

21. Van De Wiel S. Intestinal transport and signaling of bile salts: A focus on OSTa-OST $\beta .2020$

22. Vaz FM, Paulusma CC, Huidekoper $\mathrm{H}$, et al. Sodium taurocholate cotransporting polypeptide (SLC10A1) deficiency: conjugated hypercholanemia without a clear clinical phenotype. Hepatology. 2015; 61(1): 260-267, doi: 10.1002/hep.27240, indexed in Pubmed: 24867799.

23. Kojima H, Nies AT, König J, et al. Changes in the expression and localization of hepatocellular transporters and radixin in primary biliary cirrhosis. J Hepatol. 2003; 39(5):693-702, doi: 10.1016/s0168-8278(03)00410-0, indexed in Pubmed: 14568249.

24. Van Herpe F, Waterham HR, Adams CJ, et al. NTCP deficiency and persistently raised bile salts: an adult case. J Inherit Metab Dis. 2017; 40(3): 313-315, doi: 10.1007/s10545-017-0031-9, indexed in Pubmed: 28283843.

25. Sargiacomo C, El-Kehdy H, Pourcher G, et al. Age-dependent glycosylation of the sodium taurocholate cotransporter polypeptide: From fetal to adult human livers. Hepatol Commun. 2018; 2(6): 693-702, doi: 10.1002/hep4.1174, indexed in Pubmed: 29881821.

26. Shneider BL, Fox VL, Schwarz KB, et al. Hepatic basolateral sodium-dependent-bile acid transporter expression in two unusual cases of hypercholanemia and in extrahepatic biliary atresia. Hepatology. 1997; 25(5): 1176-1183, doi: 10.1002/hep.510250521, indexed in Pubmed: 9141436. 